\title{
Enhanced orientational Kerr effect in vertically aligned deformed helix ferroelectric liquid crystals
}

\author{
Evgeny P. Pozhidaev, ${ }^{1,2}$ Abhishek Kumar Srivastava, ${ }^{2, *}$ Alexei D. Kiselev ${ }^{2,3,5}$ Vladimir G. Chigrinov ${ }^{2,6}$ \\ Valery V. Vashchenko, ${ }^{4}$ Alexander I. Krivoshey, ${ }^{4}$ Maxim V. Minchenko, ${ }^{1}$ and Hoi-Sing Kwok ${ }^{2}$ \\ ${ }^{1}$ P.N. Lebedev Physical Institute of the Russian Academy of Sciences, Leninskiy pr. 53, Moscow 119991, Russia \\ ${ }^{2}$ State Key Laboratory on Advanced Displays and Optoelectronics Technologies, Department of Electronic and Computer Engineering, \\ The Hong Kong University of Science and Technology, Clear Water Bay, Kowloon, Hong Kong, China \\ ${ }^{3}$ Institute of Physics, National Academy of Sciences of Ukraine, prospekt Nauki 46, 03680 Kiev, Ukraine \\ ${ }^{4}$ State Scientific Institution "Institute for Single Crystals”, Lenin Ave., 60, Kharkov 61001, Ukraine \\ ${ }^{5}$ e-mail: kiselev@iop.kiev.ua \\ ${ }^{6}$ e-mail: eechigr@ust.hk \\ ${ }^{*}$ Corresponding author: abhishek_srivastava_lu@yahoo.com.in
}

Received February 10, 2014; revised March 21, 2014; accepted April 11, 2014;

posted April 14, 2014 (Doc. ID 206066); published May 8, 2014

\begin{abstract}
We disclose the vertically aligned deformed helix ferroelectric liquid crystal whose Kerr constant $\left(K_{\text {kerr }} \approx 130 \mathrm{~nm} / \mathrm{V}^{2}\right.$ at $\lambda=543 \mathrm{~nm}$ ) is around one order of magnitude higher than any other value previously reported for liquid crystalline structures. Under certain conditions, the phase modulation with ellipticity less than 0.05 over the range of continuous and hysteresis-free electric adjustment of the phase shift from zero to $2 \pi$ has been obtained at subkilohertz frequency. (c) 2014 Optical Society of America

OCIS codes: (160.3710) Liquid crystals; (120.5060) Phase modulation; (190.3270) Kerr effect; (230.3720) Liquid-crystal devices.

http://dx.doi.org/10.1364/OL.39.002900
\end{abstract}

Pure phase modulation of light with conserved ellipticity is in high demand for a variety of applications. These include photonic devices, such as tunable lenses, focusers, wavefront correctors and correlators [1,2], used as building blocks of optical information processors and displays. Nowadays microelectromechanical systems [3] and bimorph deformable mirrors [4] are employed for the high-frequency $(f>1 \mathrm{kHz})$ binary phase modulation with the fixed phase shift arising due to light reflection. However, the progress in the development of optical processing systems is being impeded by the lack of high performance and high-speed liquid crystal (LC) light phase modulators with continuous and hysteresis-free response [5].

Phase modulation of light based on the Kerr effect in polymer stabilized blue phase liquid crystals (PSBPLC) was recently explored in [6,7]. The largest Kerr constant, $K_{\mathrm{kerr}}$, reported for PSBPिLCs at the wavelength $\lambda=$ $514 \mathrm{~nm}$ is $33.1 \mathrm{~nm} / \mathrm{V}^{2}$ [7]. The electro-optical (EO) response time for such systems is limited to the millisecond range. Moreover, a pronounced EO hysteresis caused by the polymer network present in PSBPLC systems is not appropriate for applications.

In [8], we found that the orientational Kerr effect in a vertically aligned deformed helix ferroelectric LC (VADHFLC) with subwavelength helix pitch, $p_{0} \approx$ $150 \mathrm{~nm}$, is characterized by fast and, under certain conditions $[9,10]$, hysteresis-free electro-optics. A typical EO response time is around $100 \mu \mathrm{s}$ and is almost independent of applied electric field. Though the Kerr constant [8] $K_{\text {kerr }} \approx 27 \mathrm{~nm} / \mathrm{V}^{2}$ at $\lambda=543 \mathrm{~nm}$ is already comparable with $K_{\text {kerr }}$ of the best modern PSBPLC [7], it is feasible to increase $K_{\text {kerr }}$ of VADHFLCs further. In this Letter, we suggest an approach to enhance the Kerr effect and use it to drastically increase the Kerr constant of VADHFLC.
For the pure phase modulation, where effects of diffraction, light scattering, and polarization plane rotation of the incident light are suppressed in the visible spectral range, the selective reflection band of the VADHFLC should be in the UV region. This can be achieved when $p_{0} \ll \lambda$. For this case, the field-free $(\mathbf{E}=0)$ refractive index ellipsoid of the VADHFLC with subwavelength pitch is uniaxial [see Fig. 1 (left)], whereas applying the electric field perpendicular to the helical axis induces optical biaxiality and rotation of the optical axes [see Fig. 1 (right)] [요시].

At $E \neq 0$, the refractive indices perpendicular $\left(n_{E \perp}\right)$ and parallel $\left(n_{E \|}\right)$ to the electric field that govern propagation of normally incident light beams can be written as follows []ㅛ:

$$
n_{E \perp} / n_{p}=1+\frac{\epsilon_{e}-\epsilon_{\perp}}{\epsilon_{e}+\epsilon_{\perp}}\left[\frac{\epsilon_{0} \chi_{G}}{P_{s}}\right]^{2} E^{2}
$$

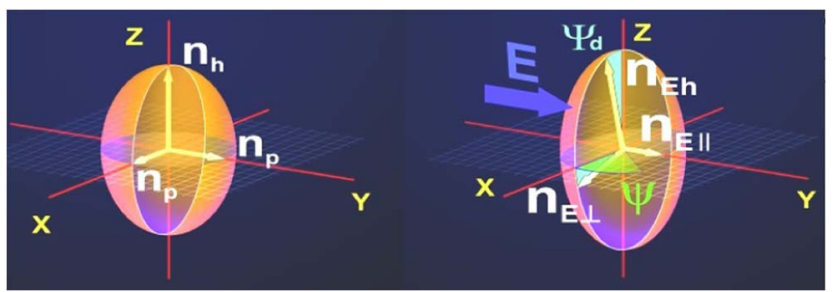

Fig. 1. Ellipsoids of effective refractive indices of a short-pitch VADHFLC cell. Left: at $\mathbf{E}=0$, the field-free effective ellipsoid is uniaxially anisotropic with the optical axis parallel to the helix axis. Right: applying an in-plane electric field $\mathbf{E} \| \hat{\mathbf{y}}$, makes the optical anisotropy biaxial with the two optical axes rotated by the angle $\Psi_{d} \propto E$ about the electric field vector $\mathbf{E}[\underline{8}, 11]$. 


$$
\begin{gathered}
n_{E \|} / n_{p}=1-\frac{\epsilon_{e}-\epsilon_{\perp}}{\epsilon_{e}+\varepsilon_{\perp}}\left[\frac{\epsilon_{0} \chi_{G}}{P_{s}}\right]^{2} E^{2} \\
n_{p}=\sqrt{\left(\epsilon_{e}+\epsilon_{\perp}\right) / 2}, \quad \epsilon_{e}=\frac{\epsilon_{\|} \epsilon_{\perp}}{\epsilon_{\perp} \sin ^{2} \theta+\epsilon_{\|} \cos ^{2} \theta},
\end{gathered}
$$

where $\epsilon_{\|}\left(\epsilon_{\perp}\right)$ is the high-frequency dielectric constant measured parallel (perpendicular) to the FLC director; $\theta$ is the smectic tilt angle; $P_{s}$ is the spontaneous ferroelectric polarization, and $\epsilon_{0}$ is the dielectric permittivity of free space. The dielectric susceptibility of the Goldstone mode, $\chi_{G}$, is given by [트]

$$
\chi_{G}=\epsilon_{0}^{-1} \frac{\partial P}{\partial E}=\frac{P_{s}^{2}}{2 \epsilon_{0} K q_{0}^{2} \sin ^{2} \theta},
$$

where $q_{0}=2 \pi / p_{0}$ and $K$ is the effective twist elastic constant

From Eqs. (1)-(4), the field-induced in-plane birefringence

$$
\delta n_{i}=n_{E \perp}-n_{E \|}=2 n_{p} \frac{\epsilon_{e}-\epsilon_{\perp}}{\epsilon_{e}+\epsilon_{\perp}}\left[\frac{\epsilon_{0} \chi_{G}}{P_{s}}\right]^{2} E^{2}=K_{\mathrm{kerr}} \lambda E^{2}
$$

can be expressed in terms of the Kerr constant given by

$$
K_{\mathrm{kerr}}=\frac{n_{p}}{\lambda} \frac{\epsilon_{e}-\epsilon_{\perp}}{\epsilon_{e}+\epsilon_{\perp}} \frac{P_{s}^{2} p_{0}^{4}}{32 \pi^{2} K^{2} \sin ^{4} \theta}
$$

From Eq. (6) it is clear that an increase in $P_{s}$ and $p_{0}$ will enhance the Kerr constant $K_{\text {kerr. }}$ There are, however, certain limitations on these parameters: (a) the pitch $p_{0}$ should be sufficiently small so as to avoid the effects of selective reflection band mentioned above and (b) an increase in $P_{s}$ of a chosen FLC mixture requires high concentrations of chiral molecules that will increase the rotational viscosity of the mixture $\gamma_{\phi}$, thus affecting the EO response time $[13,14]$. So, there is a trade-off between high value of $\overline{K_{\mathrm{kerr}}}$ and fast $\mathrm{EO}$ response. In our experiments, the optimal relationship between the parameters $\gamma_{\phi}, \theta, K, P_{s}$, and $p_{0}$ leading to a drastic increase of $K_{\text {kerr }}$ has been achieved with a proper choice of specially selected chemical structures of the FLC mixture components and their concentrations.

The method of mixing achiral smectic $\mathrm{C}$ and chiral nonmesogenic compound [15] was employed to elaborate a FLC mixture that meets the above constraints. A peculiarity of the mixture is that it contains two chiral nonmesogenic compounds, which induce in achiral smectic C matrix spontaneous polarization of the same sign, whereas their optical twisting powers are opposite in sign [16]. This enables the relation between $P_{s}$ and $p_{0}$ to be fine tuned while the value of $P_{s}$ increases.

One of these compounds is a fluorinated derivative of the p-terphenyldicarboxylic acid that exhibit extremely high twisting power [17] (see compound A in Fig. 2). Another chiral compound is a lactate derivative of the p-terphenyldicarboxylic acid shown as compound B in
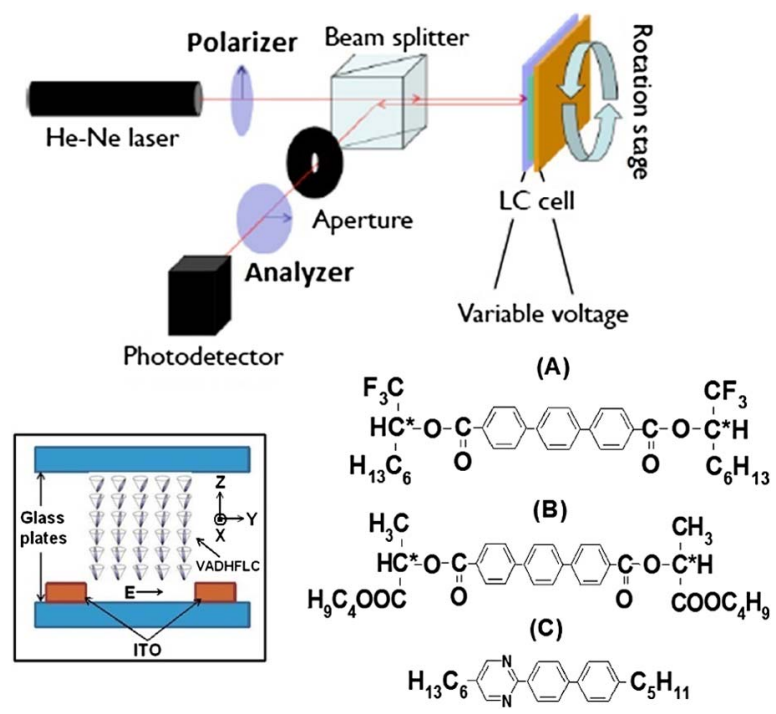

Fig. 2. Top: experimental setup for reflectance measurements. Glan prisms were used as both polarizer and analyzer. Bottom (left): VADHFLC cell with ITO electrodes (the width is $20 \mu \mathrm{m}$ and the interelectrode gap is $100 \mu \mathrm{m}$ ) and the axes of the coordinate system shown in Fig. 1. Bottom (A)-(C): chemical structures of compounds used to prepare the FLC-618.

Fig. 2. Achiral smectic $\mathrm{C}$ matrix of the mixture is the biphenylpirimidine indicated as compound $\mathrm{C}$ in Fig. $\underline{2}$.

The eutectic mixture of these compounds corresponds to the following weight concentrations: $39 \%$ of compound A, $9 \%$ of compound $\mathrm{B}$, and $52 \%$ of compound $\mathrm{C}$. The mixture is named FLC-618 and is characterized by $p_{0}=175 \mathrm{~nm}$ and $P_{s}=200 \mathrm{nC} / \mathrm{cm}^{2}$ (at temperature $T=22^{\circ} \mathrm{C}$ ). The phase transitions sequence of this FLC at heating from preliminary obtained solid crystalline state is $\mathrm{Cr} \stackrel{+21^{\circ} \mathrm{C}}{\longrightarrow} \mathrm{Sm} C \stackrel{\star+100^{\circ} \mathrm{C}}{\longrightarrow} \mathrm{Sm} A^{\star} \stackrel{+117^{\circ} \mathrm{C}}{\longrightarrow}$ Iso, whereas at cooling down from isotropic phase crystallization occurs around $+6^{\circ} \mathrm{C}$.

EO studies have been carried out in the reflective geometry shown in Fig. 2 with a VADHFLC layer of thickness $d_{\mathrm{FLC}}=18 \mu \mathrm{m}$ placed between either crossed or parallel polarizers at $\Psi=45^{\circ}$ and $\Psi=0$, where $\Psi$ angle is the angle between the polarization plane of incident light and the $X$ axis normal to the electric field (see Fig. 1). A helium-neon laser with wavelength either 632.8 or $543 \mathrm{~nm}$ was used as a source of light. The EO response of a VADHFLC cell at $E=1 \mathrm{~V} / \mu \mathrm{m}$ shown in the insert of Fig. 3 confirms good optical quality (the contrast ratio is better than 1000:1) and the response time around $300 \mu \mathrm{s}$.

When polarizer and analyzer are parallel and $E=0$, the intensity of light, $I_{\|}^{E=0}$, that after all reflections (from dielectric surfaces of the cell, beam splitter, and Glan prisms used as the polarizers) is collected by the photodetector, is proportional to the laser beam intensity, $I_{0}$ : $I_{\|}^{E=0}=r I_{0}$. In the case of crossed polarizers with $\Psi \neq 0$ and $E \neq 0$, the light intensity collected by the photodetector $I_{\perp}^{E \neq 0}$ can be conveniently expressed in terms of the normalized reflectance given by

$$
R=I_{\perp}^{E \neq 0} / I_{\|}^{E=0}=\sin ^{2}(2 \Psi) \sin ^{2} \frac{2 \pi \delta n_{i} d_{\mathrm{FLC}}}{\lambda}
$$




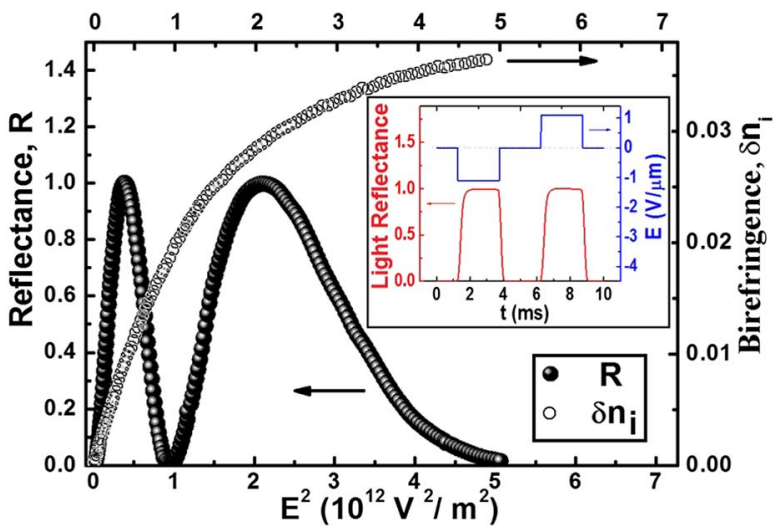

Fig. 3. Dependence of the light reflectance of a $18 \mu \mathrm{m}$ thick VADHFLC cell on square of electric field $E^{2}$ (filled circles). Measurements were carried out in the reflective mode with crossed polarizer and analyzer at temperature $55^{\circ} \mathrm{C}$, wavelength $\lambda=632.8 \mathrm{~nm}$, EO response frequency $500 \mathrm{~Hz}$, and $\Psi=45^{\circ}$. Electric field-induced birefringence $\delta n_{i}$ (open circles) was evaluated as a function of $E^{2}$ by using Eq. (7). Insert: EO response of the VADHFLC cell (red curve at the bottom) under the applied alternating signal (blue curve on the top).

where $\delta n_{i}$ plays the role of field-induced in-plane birefringence for a light beam propagating along the normal to the cell (the $Z$ axis).

Formula (7) can now be used to evaluate $\delta n_{i}$ from the measured reflectance $R$. Typical results for the electric field dependencies of $R$ and $\delta n_{i}$ are presented in Fig. 3 . In the reflective mode, the phase retardation resulted from the electrically induced biaxiality can be expressed in terms of the Kerr constant as follows:

$$
\Delta \Phi_{\mathrm{ret}}=\frac{4 \pi \delta n_{i} d_{\mathrm{FLC}}}{\lambda}=4 \pi K_{\mathrm{kerr}} E^{2} d_{\mathrm{FLC}}
$$

Referring to Fig. $\underline{3}$, it is clear that, for $\Psi=45^{\circ}, \Delta \Phi_{\text {ret }}=$ $4 \pi$ at $E \approx 2.3 \mathrm{~V} / \mu \mathrm{m}$. It can also be seen that, owing to rapidly growing higher-order nonlinearities, the Kerr-like dependence (5) is no longer valid at the phase retardation above $2 \pi(E \geq 1 \mathrm{~V} / \mu \mathrm{m})$.

The curves representing the Kerr constant, $K_{\text {kerr }}$, evaluated at different temperatures are plotted in Fig. $\underline{4}$. It is seen that $K_{\text {kerr }}$ initially increases with temperature, reaches the maxima, and decreases at higher temperatures. This behavior of $K_{\text {kerr }}$ can be explained by the temperature dependence of $P_{s}$ and $p_{0}$ that enter Eq. (6). The largest value of $K_{\text {kerr }}$ evaluated for our VADHFLC is $130 \mathrm{~nm} / \mathrm{V}^{2}$.

In order to demonstrate how relation (6) may guide the way to enhance the Kerr constant using classical methods of FLC material science, we compare the results for two FLC mixtures: the newly developed FLC-618 and the mixture FLC-587 described in [8]. The parameters of FLC-587 at temperature $60^{\circ} \mathrm{C}$ are: $\bar{\theta}=35^{\circ}, p_{0}=210 \mathrm{~nm}$, $P_{s}=110 \mathrm{nC} / \mathrm{cm}^{2}$. Similarly, for FLC- 618 at $T=60^{\circ} \mathrm{C}$ we have: $\theta=33^{\circ}, p_{0}=250 \mathrm{~nm}$ (see insert in Fig. 5), $P_{s}=160 \mathrm{nC} / \mathrm{cm}^{2}$. These parameters combined with Eq. (6) can now be used to obtain a theoretical estimate for the Kerr constants ratio $K_{\mathrm{kerr}}^{\mathrm{FLC}-618} / K_{\mathrm{kerr}}^{\mathrm{FLC}-587} \approx 5.2$. This

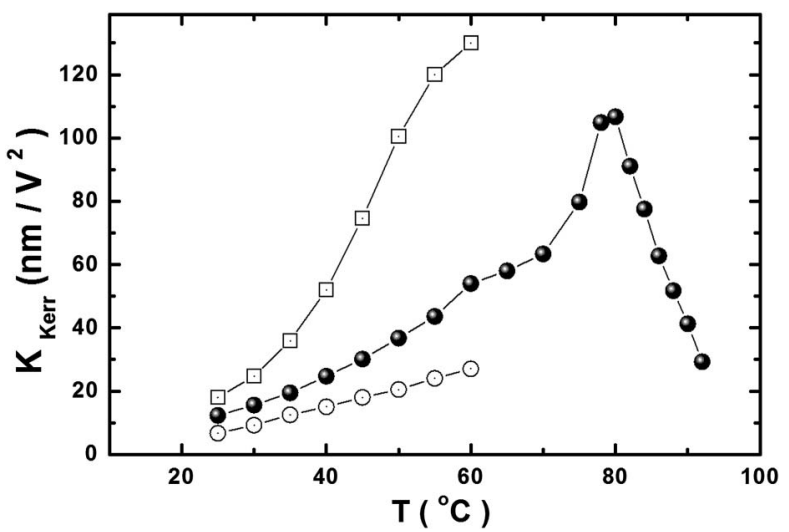

Fig. 4. Temperature dependencies of Kerr constant measured in a FLC-618 cell at $\lambda=632.8 \mathrm{~nm}$ (filled circles) and at $\lambda=$ $543 \mathrm{~nm}$ (open squares). Open circles represent the data measured in a FLC-587 cell at $\lambda=543 \mathrm{~nm}$ [ㅇ] .

estimate agrees reasonably well with the experimental value of this ratio, which is about 4.8 (see Fig. 4).

It should be emphasized that, at $\Psi=45^{\circ}$, when the phase retardation (8) continuously changes between 0 and $4 \pi$, the ellipticity of reflected light significantly varies. This was checked experimentally using the known polarimetry method [18]. In other words, the phase retardation arises from the electrically controlled birefringence (5) so that both amplitude and phase modulation of light are observed. This geometry of VADHFLC is more appropriate for display devices and, as compared to other alternatives, has the important advantage of large $K_{\text {kerr }}$.

At $\Psi=0$, the polarization plane of incident light is parallel to the plane where the optical axes lie (the $X-Z$ plane in Fig. 1) and the incident light beam does not split into ordinary and extraordinary rays. In this case, similar to B-effect in nematic LCs, the phase shift $\Delta \Phi_{\perp}$ of incident light occurs solely due to changes in the refractive index $n_{E \perp}$. This is the regime of pure phase modulation. It is not difficult to see that the phase shift at $\Psi=0$ is equal to one-half the phase retardation at $\Psi=45^{\circ}$ :

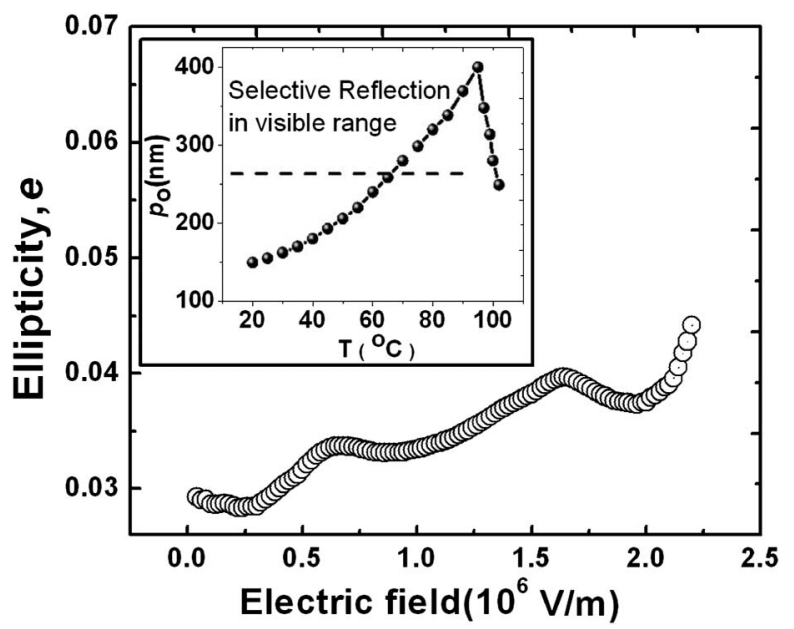

Fig. 5. Ellipticity of light reflected from a $18 \mu \mathrm{m}$ thick VADHFLC cell versus applied electric field measured at $\Psi=0, f=500 \mathrm{~Hz}, T=55^{\circ} \mathrm{C}$, and $\lambda=632.8 \mathrm{~nm}$. Insert: temperature dependence of the helix pitch for FLC-618. Selective reflection is observed when the pitch is above the dashed line. 


$$
\Delta \Phi_{\perp}=\frac{4 \pi\left(n_{E \perp}-n_{p}\right) d_{\mathrm{FLC}}}{\lambda}=\frac{1}{2} \Delta \Phi_{\mathrm{ret}} .
$$

So, at $\Psi=0$ and $E=2.3 \mathrm{~V} / \mu \mathrm{m}$, we have the $2 \pi$ phase shift.

Referring to Fig. 5 , when the phase shift $\Delta \Phi_{\perp}$ changes between 0 and $2 \pi$, variations of the ellipticity are ranged from 0.027 to 0.045 . Experimental imperfections and small fluctuations in orientation of the optical axes that are neglected in the theory [8] can be attributed to these variations. For practical applications, this can be regarded as a subkilohertz pure phase modulation of light with the conserved ellipticity.

In conclusion, based on the theoretical analysis and material optimization, we revealed an efficient strategy to enhance the Kerr effect in VADHFLCs and have achieved the large value of the Kerr constant, $K_{\text {kerr }} \approx 130 \mathrm{~nm} / \mathrm{V}^{2}$. Further improvements in material optimization for $P_{s}$ and $p_{0}$ of the FLC material are possible to accomplish the pure $2 \pi$ phase modulation at room temperature. The proposed VADHFLC systems with a continuous, hysteresis-free $2 \pi$ phase modulation at subkilohertz frequencies could find application in many modern photonic and display devices demanding fast phase only modulation at low electric fields.

This work is supported by the HKUST grants CERG 612310 and RGC 614410 and by RFBR grants 13-0200598_A, 1302-90487_Ukr_f_a.

\section{References}

1. S. Xu, H. Ren, and S.-T. Wu, Opt. Express 20, 28518 (2012).

2. L. Hu, L. Xuan, Y. Liu, Z. Cao, D. Li, and Q. Mu, Opt. Express 12, 6403 (2004).
3. M. C. Roggeman, V. M. Bright, B. M. Welsh, S. R. Hick, P. C. Roberts, W. D. Cowan, and J. H. Comtois, Opt. Eng. 36, 1326 (1997).

4. N. T. Adelman, Appl. Opt. 16, 3075 (1977).

5. V. Shrauger and C. Warde, Proc. SPIE 4291, 101 (2001).

6. Y. Hisakado, H. Kikuchi, T. Nagamura, and T. Kajiyama, Adv. Mater. 17, 96 (2005).

7. Y. Chen, D. Xu, S.-T. Wu, S.-i. Yamamoto, and Y. Haseba, Appl. Phys. Lett. 102, 141116 (2013).

8. E. P. Pozhidaev, A. D. Kiselev, A. K. Srivastava, V. G. Chigrinov, H.-S. Kwok, and M. V. Minchenko, Phys. Rev. E 87, 052502 (2013).

9. L. M. Blinov, S. P. Palto, E. P. Pozhidaev, Y. P. Bobylev, V. M. Shoshin, A. L. Andreev, F. V. Podgornov, and W. Haase, Phys. Rev. E 71, 071715 (2005).

10. E. Pozhidaev, V. Chigrinov, A. Murauski, V. Molkin, D. Tao, and H.-S. Kwok, J. Soc. Inf. Disp. 20, 273 (2012).

11. A. D. Kiselev, E. P. Pozhidaev, V. G. Chigrinov, and H.-S. Kwok, Phys. Rev. E 83, 031703 (2011).

12. B. Urbanc, B. Žekš, and T. Carlsson, Ferroelectrics 113, 219 (1991).

13. M. I. Barnik, V. A. Baikalov, V. G. Chigrinov, and E. P. Pozhidaev, Mol. Cryst. Liq. Cryst. 143, 101 (1987).

14. E. P. Pozhidaev, M. A. Osipov, V. G. Chigrinov, V. A. Baikalov, L. M. Blinov, and L. A. Beresnev, Zh. Eksp. Teor. Fiz. 94, 125 (1988).

15. W. Kuczynski and H. Stegemeyer, Chem. Phys. Lett. 70, 123 (1980).

16. A. Z. Rabinovich, M. V. Loseva, N. I. Chernova, E. P. Pozhidaev, O. S. Petrashevich, and J. S. Narkevich, Liq. Cryst. 6, 533 (1989).

17. E. P. Pozhidaev, S. I. Torgova, V. E. Molkin, M. V. Minchenko, V. V. Vashchenko, A. I. Krivoshey, and A. Strigazzi, Mol. Cryst. Liq. Cryst. 509, 1042 (2009).

18. A. D. Kiselev, R. G. Vovk, R. I. Egorov, and V. G. Chigrinov, Phys. Rev. A 78, 033815 (2008). 